\title{
ESTUDIO ETNOZOOLÓGICO DEL USO TRADICIONAL DE AVES SILVESTRES EN LA COMUNIDAD MAYA DE XANLÁH, YUCATÁN, MÉXICO
}

\author{
ETNOZOOLOGICAL STUDY OF TRADITIONAL USE OF WILD BIRDS IN THE MAYAN \\ COMMUNITY OF XANLAH, YUCATAN, MEXICO
}

Yeni Ruth CHONTAL CHAGALA ${ }^{1}$; Wilian de Jesús AGUILAR CORDERO ${ }^{2 *}$; Juan CHABLÉ SANTOS ${ }^{1}$

${ }^{1}$ Departamento de Zoología, ${ }^{2}$ Departamento de Botánica. Campus de Ciencias Biológicas y Agropecurias. Licenciatura en Biología. Universidad Autónoma de Yucatán, México; *凶 acordero@correo.uady.mx

\begin{abstract}
Based on the various interactions in the history between humans and wild birds, they have been an important resource in the social and cultural development of human societies. The use of wild birds in the Mayan community of Xanlah, Chankom, Yucatan was characterized in this study. The data were collected from January to June 2015 through mixed questionnaires from 55 people of both genders and 5 semi-structured interviews with local knowers. 27 species of birds were recorded in 18 families and 12 taxonomic orders, which are used under eight categories of use: food, forecaster, medicinal, pet, mythical, tool, ornamental and story. The species that obtained a high value of use (IVU) were Ortalis vetula (Wagler, 1830) with 0.65, followed by Meleagris ocellata (Cuvier, 1820) with 0.49 and Tyto alba (Scopoli, 1769) with 0.41. With respect to the significant usage level (UST) four species had a value of more than $20 \%$. The results demonstrate the continuity in the belief system and in the biocultural memory of the Yucatan Mayan communities in the relationship between society/nature.

Keywords: Biocultural knowledge, ethnoornithology, level of significant use of TRAMIL, Mayan communities, Use Value (UV's).
\end{abstract}

Submitted: 07/09/2019; Accepted: 04/11/2019

\section{RESUMEN}

A partir de las diversas interacciones en la historia entre el ser humano y las aves, estas han sido recurso importante en el desarrollo social y cultural de las sociedades humanas. En el presente estúdio se caracterizó el uso de las aves silvestres en la comunidad maya de Xanláh, Chankom, Yucatán. Los datos fueron recolectados de enero a junio de 2015 por medio de cuestionarios mixtos realizados a 55 pobladores de ambos géneros y 5 entrevistas semi-estructuradas a sabedores locales. Se registraron 27 especies de aves distribuidas en 18 familias y 12 órdenes taxonómicos, las cuales fueron ubicados en ocho categorías de uso: alimento, pronosticador, medicinal, mascota, mítico, herramienta, ornamental y relato. Las especies que obtuvieron un alto valor de uso (IVU) fueron Ortalis vetula (Wagler, 1830) con 0.65 , seguido por Meleagris ocellata (Cuvier, 1820) con 0.49 y Tyto alba (Scopoli, 1769) con 0.41 . Con respecto al nivel de uso significativo (UST) cuatro especies presentaron un valor superior al $20 \%$. Los resultados evidencian la continuidad en el sistema de creencias y en la memoria biocultural de las comunidades mayas yucatecas en la relación sociedad/naturaleza.

Palabras-clave: conocimiento biocultural, etno-ornitología, nível de uso significativo TRAMIL, comunidad maya, índice de valor de uso.

\section{INTRODUCCIÓN}

En México, desde la época prehispánica, existen numerosas manifestaciones del aprecio e interés hacia las aves, constituyéndose como recurso importante en el desarrollo social y cultural de las sociedades humanas a través de sus usos para diversos fines, entre los que destacan el alimento, medicina, comercio, ornamental o simplemente por el placer de observar su plumaje y escuchar su canto (LÓPEZ-MEDELLÍN e ÍÑIGO, 2009; CHABLÉ y DELFÍN, 2010; NARANJO et al., 2010).

La estrecha relación hombre-naturaleza ha creado múltiples asociaciones con los recursos silvestres ubicando a los pobladores indígenas como actores clave, ya que forman parte de la sabiduría tradicional y de la cultura de una región (GONZÁLEZ, 2001).

Las comunidades rurales han adquirido a través de su dependencia, una cantidad inmensa de 
conocimiento de su entorno general, principalmente a través de su relación con la naturaleza y estilos de vida, realizando un proceso que ha llevado a que cada especie animal tenga un uso práctico, un sentido sagrado o un recuerdo individual o colectivo (ANTONIO, 2012).

Este conjunto de conocimientos, prácticas y creencias, representan la experiencia de múltiples generaciones que son transmitidas a través de diversos procesos de enseñanza-aprendizaje y que son producto de un proceso acumulativo y dinámico de experiencias prácticas y adaptación al cambio (REYES-GARCÍA, 2009; UC, 2014).

Una forma de aproximación al estudio de las interacciones de las comunidades rurales y los animales es por medio de la etnozoología, caracterizada como una etnociencia que busca comprender cómo las diferentes culturas perciben e interactúan con los recursos faunísticos con los que conviven (ALVES y SOUTO, 2015). Por ejemplo, García et al. (2017) en un estudio realizado en comunidad de Zacualpan de Amilpas, Morelos, México se analizó el conocimiento tradicional de los habitantes originarios con relación a las aves y mamíferos silvestres que se asocian a los huertos frutícolas tradicionales donde se concluyó que las personas conservan el conocimiento etnozoológico de las aves y mamíferos, porque pueden reconocer y nombrar a las diversas especies que llegan a sus huertos.

En el estado de Campeche, México, los pobladores satisfacen sus requerimientos materiales y/o culturales a través del uso directo e indirecto de la fauna silvestre, como lo señalan PUC-GIL y Retana-Guiascón (2012) en su estudio realizado en la comunidad de Villa de Guadalupe (Champotón), donde se documentó el conocimiento tradicional relacionado con el uso de aves bajo cinco categorías: alimenticio, medicinal, mascota, mítico y herramienta. Destacando principalmente, el pavo de monte (Meleagris ocellata), la perdiz (Crypturellus cinnamomeus) y la chachalaca (Ortalis vetula), ya que éstas son las de mayor tradición de caza entre las comunidades mayas. Asimismo, registraron una mayor preferencia por loros y palomas como mascotas y comerciales.

En el caso de Yucatán, son escazas las investigaciones etno-ornitológicas actuales (UC y CERVERA, 2014; GONZALEZ et al., 2018), aunque en el caso arqueológico hay más reportes de representaciones simbólicas de las aves en el mundo maya prehispánico y en los códices mayas (TOZZER y ALLEN, 1910; DE LA GARZA, 1995; NAVARIJO, 2001; GÖTZ, 2012). Es por lo antes señalado, que en el presente estudio se contribuye a la generación y documentación del conocimiento tradicional del pueblo maya yucateco sobre la avifauna, a través de la caracterización de los usos y preferencias sobre las aves silvestres en la comunidad maya de Xanláh, Yucatán, México.

\section{MATERIALES Y MÉTODOS}

\section{Área de estudio}

El estudio se realizó en la comunidad maya de Xanláh, localizado en el municipio de Chankom, en el estado de Yucatán, México. Está comprendido entre las coordenadas geográficas $20^{\circ}$ 31' 02" de latitud norte y $88^{\circ} 29^{\prime} 36^{\prime \prime}$ de longitud oeste (Figura 1).
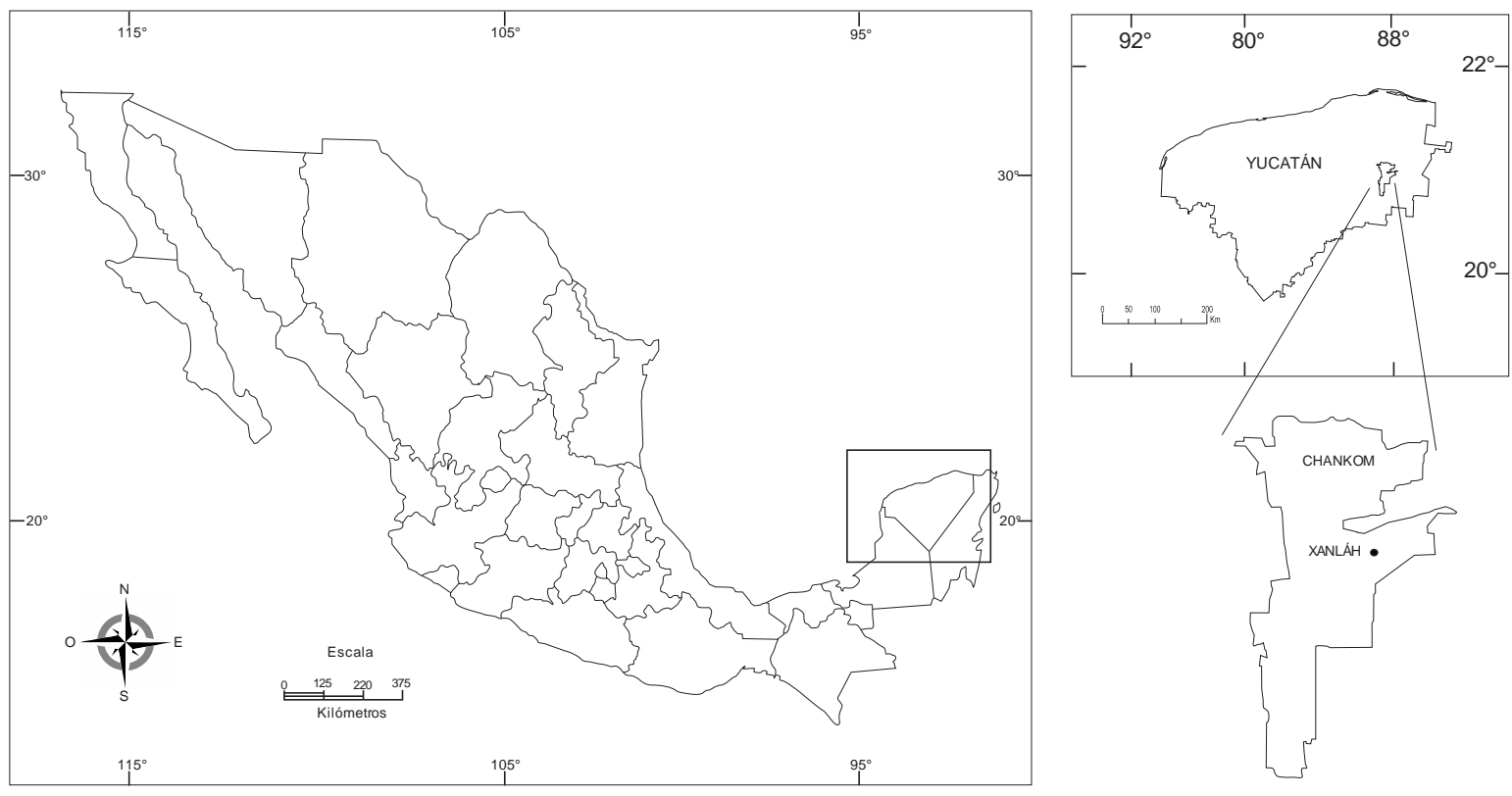

Figura 1. Ubicación geográfica de Xanláh, Yucatán, México. (Fuente: INEGI) 
El trabajo de campo se realizó durante los meses de febrero a junio de 2015 en un período de cinco estancias en la comunidad de Xanláh con una duración de una semana cada una.

En el proceso de investigación etnobiológica es fundamental buscar establecer el rapport que consiste en crear un vínculo de confianza, empatía y cercanía con las personas (MORGA, 2012; BALBUENA, 2017). Es por esto que se realizó en una primera estancia de campo, una reunión con las autoridades formales de la comunidad, el comisario ejidal y municipal, para explicar el trabajo y solicitar su permiso, asimismo durante esta estancia se aplicó la prueba piloto para validar el cuestionario con el fin de identificar y eliminar cualquier problema en la estructura de este mismo.

Para la investigación de campo y recolecta de datos, se diseñó un cuestionario mixto y se aplicó a través de una encuesta a la población. También se diseñó y aplicó entrevistas semiestructuradas a sabedores locales (AGUIRRE, 1995; RUSELL, 1995; GUBER, 2005).

Otra técnica cualitativa que se usó es la señalada por Ferro (2010) como observación etnográfica, que permitió registrar en el diario de campo información del comportamiento de los individuos hacia el uso y aprovechamiento de las aves, así como aspectos de la cotidianidad.

El cuestionario se aplicó en cada hogar visitado, dirigidos a las cabezas de familia de la comunidad, el cual incluía los siguientes dimensiones o apartados: a) datos generales b) usos de las aves, c) partes usadas de las aves, d) especies consideradas benéficas o dañinas y e) especies utilizadas en ceremonias o asociadas a alguna leyenda o mito. Durante la aplicación de los cuestionarios se identificaron las especies de aves (Figura 2) a través de la consulta a un catálogo ilustrado con imágenes de aves de la región obtenidas de las guías ornitológicas de Howell y Web (1995), Peterson y Chalif (1989), Dunn y Alderfer (2008), Sibley (2000) y Kaufman (2005), así como recursos auditivos (cantos de aves) del portal https://www.xeno-canto.org/.

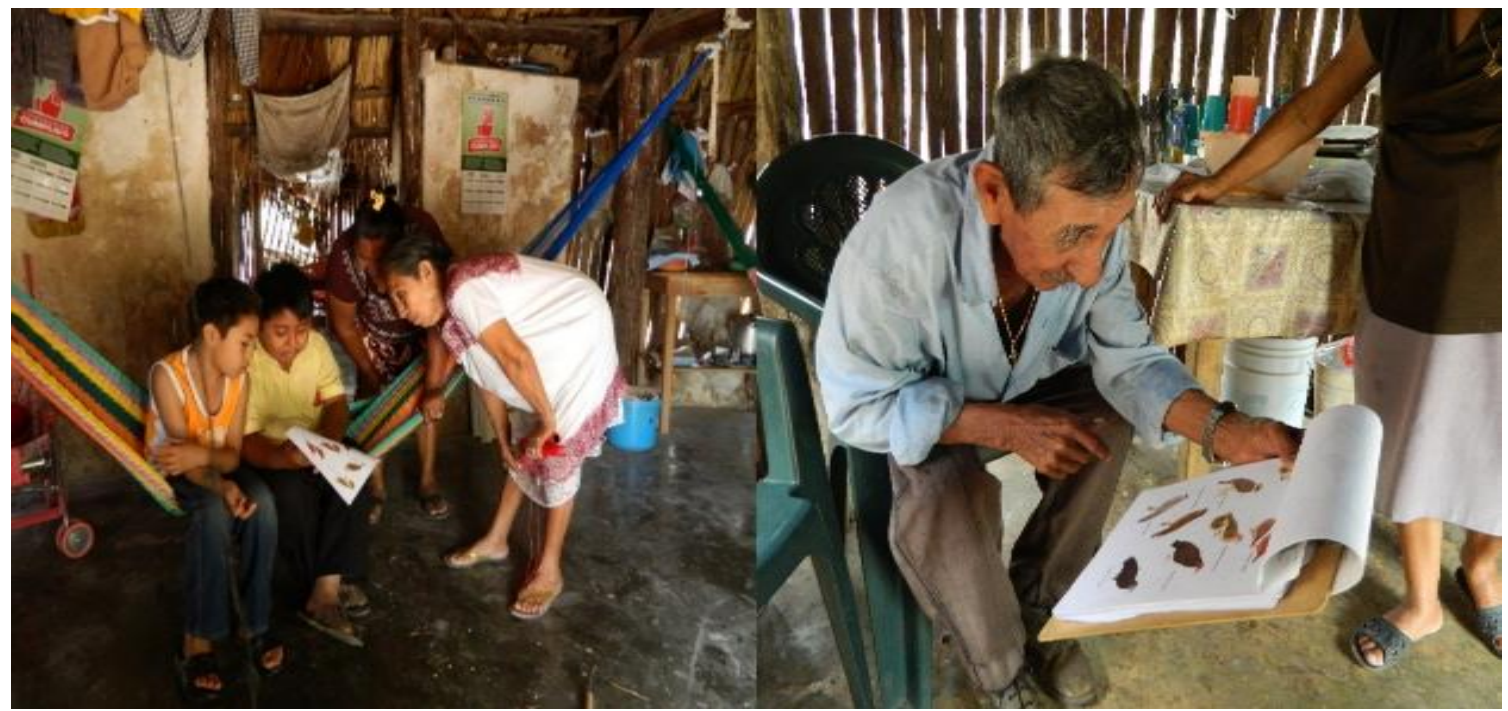

Figura 2. Identificación de las aves con fichas ilustradas empleadas en la comunidad de Xanláh, Yucatán.

Se aplicó entrevistas semiestructuradas a sabedores locales para ampliar la narrativa relacionada con los conocimientos de usos y relatos que no fueron profundizados a través del dato cuantitativo del cuestionario. Por otro lado, antes de la aplicación de las entrevistas se buscó establecer el rapport con los entrevistados, con la finalidad de garantizar que la obtención de la información sea lo más cercana a la realidad. Para la aplicación de las entrevistas se seleccionaron personas con base en los siguientes criterios: a) personas con conocimiento sobre el uso de aves, b) disponibilidad al diálogo, c) que habiten dentro o en las cercanías de la comunidad y d) que sean personas apreciadas por la comunidad. Las entrevistas fueron grabadas con previa autorización de los entrevistados, mediante una grabadora de voz, para posteriormente ser transcritas con el Software F4 Transcription y ser analizadas y usadas en la redacción de los resultados.

Los datos obtenidos a través de los cuestionarios fueron capturados, organizados y sistematizados en una base de datos en Microsoft Office Excel (2007), para realizar el análisis estadístico descriptivo. Además, se obtuvo el índice de valor de uso (IVU) usado por Toscano (2006), para estimar la importancia del valor de uso de cada especie de ave para todos los encuestados, utilizando la siguiente fórmula: 
Dónde:

UVis: es el número de usos mencionados por cada informante (i), para cada especie (s). $n_{s}$ : Número de informantes.

A su vez, se estimó el nivel de uso significativo (UST), propuesta por Germosén-Robineau (1995, apud TOSCANO, 2006) utilizando la siguiente formula:

Dónde:

$$
\text { UST }=\frac{\text { Uso Especie }(s)}{\text { nis }} x 100
$$

Uso Especie (s), es el número de citas para cada especie.

nis, es el número de informantes.

Este índice, según Toscano (2006) expresa que aquellos usos que son citados con una frecuencia superior o igual al $20 \%$, pueden considerarse significativos desde el punto de vista de su aceptación cultural y por consiguiente suponer que todavía existe un proceso de fortalecimiento del conocimiento tradicional.

A pesar de que dichos índices han sido empleados para estudios botánicos también han sido aplicados para estudios faunísticos (ALVES et al., 2012; BARROS et al., 2012; PUC y RETANAGUIASCÓN, 2012).

Posterior al análisis de datos, se identificaron las diferentes especies, reconociendo los principales usos que la comunidad hace de las aves. De manera que se generó una categorización de usos agrupadas de la siguiente manera:

a) Uso alimenticio: incluye a todas las aves que son cazadas para el aprovechamiento de su carne con fines comestibles.

b) Uso herramienta: se agruparon las aves que son complementarias o sustitutos para la ejecución de labores en el hogar o el campo.

c) Uso mascota: principalmente integrada por aves de compañía.

d) Uso medicinal: aves que son utilizadas para aliviar padecimientos o enfermedades de origen natural y cultural.

e) Uso ornamental: aquellos derivados de las aves que son expuestas en partes visibles de la vivienda.

f) Uso pronosticador: en esta categoría se agruparon a las aves que presagian eventos negativos o positivos para la comunidad, generalmente suelen ser denominadas aves de augurio (término que proviene del latín augurius que significa "oír a las aves). También se consideraron a las aves bioindicadoras de eventos climáticos.

g) Uso relato: se consideraron las aves que fueron nombradas en historias relacionadas con la percepción de la naturaleza.

Este estudio fue desarrollado con base a los lineamientos del Comité de Bioética del Campus de Ciencias Biológicas y Agropecuarias de la Universidad Autónoma de Yucatán, México. Además, toda la información producto de los cuestionarios y entrevistas fue de forma voluntaria.

\section{RESULTADOS}

\section{Datos sociodemográficos}

Se aplicaron un total de 55 cuestionarios mixtos a la población maya-yucateca; 29 se aplicaron a mujeres y 26 a hombres, de los cuales cinco fueron entrevistados. En promedio, los pobladores tuvieron una edad de 45 años, con una edad mínima de 16 años y una máxima de 86 . El $27 \%$ de los encuestados no poseen estudios formales, $16 \%$ cuenta con primaria incomplenta, $24 \%$ primaria completa, $4 \%$ secundaria incompleta, $25 \%$ secundaria completa y el $2 \%$ educación superior. El caso de la ocupación principal el $31 \%$ es campesino-milpero, el $6 \%$ es apicultor, el $11 \%$ empleado, el $45 \%$ ama de casa y el $7 \%$ otras actividades. El $80 \%$ de los encuestados están casados. 


\section{Uso actual de la avifauna}

La comunidad de Xanláh utiliza 27 especies de aves de 18 familias y 12 órdenes taxonómicos; siendo los representantes de las familias Cracidae, Phasianidae y Columbidae los más usados (Tabla $1)$.

Tabla 1. Aves usadas por los pobladores de la comunidad de Xanláh, Yucatán. Uso: A=Alimento, $\mathrm{He}=$ Herramienta, $\mathrm{Ma}=$ Mascota, $\mathrm{Me}=$ Medicinal, $\mathrm{O}=$ Ornamental, Pro= Pronosticador, $\mathrm{R}=$ Relato .

\begin{tabular}{|c|c|c|}
\hline FAMÍLIA - ESPECIE & NOMBRE COMÚN & TIPOS DE USOS \\
\hline \multicolumn{3}{|l|}{ TINANIFORMES } \\
\hline Crypturellus cinnamomeus & Perdiz & A \\
\hline \multicolumn{3}{|l|}{ GALLIFORMES } \\
\hline Ortalis vetula & Chachalaca & A, Me, Pro \\
\hline Colinus nigrogularis & Codorniz yucateca & A \\
\hline Dactylortyx thoracicus & Codorniz silbadora & A \\
\hline Meleagris ocellata & Pavo de monte & $\mathrm{A}, \mathrm{C}, \mathrm{He}, \mathrm{O}$ \\
\hline \multicolumn{3}{|l|}{ CATHARTIFORMES } \\
\hline Coragyps atratus & Zopilote cabeza negra & Pro \\
\hline \multicolumn{3}{|l|}{ FALCONIFORMES } \\
\hline Herpetotheres cachinnans & Halcón guaco & Pro \\
\hline \multicolumn{3}{|l|}{ COLUMBIFORMES } \\
\hline Columba livia & Paloma doméstica & Ma \\
\hline Patagioenas flavirostris & Paloma morada & $\mathrm{A}, \mathrm{Ma}, \mathrm{Me}$ \\
\hline Zenaida asiatica & Paloma ala blanca & $\mathrm{A}, \mathrm{Ma}, \mathrm{Me}$ \\
\hline Columbina talpacoti & Tórtola rojiza & A \\
\hline \multicolumn{3}{|l|}{ PSITTACIFORMES } \\
\hline Amazona albifrons & Loro frente blanca & Ma \\
\hline Amazona xantholora & Loro yucateco & $\mathrm{Me}$ \\
\hline \multicolumn{3}{|l|}{ CUCULIFORMES } \\
\hline Geococcyx velox & Correcaminos tropical & Pro \\
\hline Crotophaga sulcirostris & Garrapatero pijuy & Pro, R \\
\hline \multicolumn{3}{|l|}{ STRIGIFORMES } \\
\hline Tyto alba & Lechuza de campanario & Pro \\
\hline Megascops guatemalae & Tecolote vermiculado & Pro \\
\hline Glaucidium brasilianum & Tecolotito bajeño & Pro \\
\hline Ciccaba virgata & Búho café & Pro \\
\hline \multicolumn{3}{|l|}{ CAPRIMULGIFORMES } \\
\hline Nyctidromus albicollis & Tapacaminos pauraque & $\mathrm{C}$ \\
\hline \multicolumn{3}{|l|}{ APODIFORMES } \\
\hline Amazilia sp. & Colibrí & $\mathrm{Me}$ \\
\hline \multicolumn{3}{|l|}{ CORACIIFORMES } \\
\hline Eumomota superciliosa & Momoto corona azul & $\mathrm{Me}$ \\
\hline \multicolumn{3}{|l|}{ PASSERIFORMES } \\
\hline Cyanocorax yucatanicus & Chara yucateca & $\mathrm{Me}$ \\
\hline Cyclarhis gujanensis & Vireo ceja rufa & Pro \\
\hline Stelgidopteryx ridgwayi & Golondrina aliserrada & Pro \\
\hline Dives dives & Tordo cantor & Pro, $\mathrm{Me}$ \\
\hline Icterus gularis & Bolsero de Altamira & Pro \\
\hline
\end{tabular}




\section{Uso alimenticio}

En esta categoría se ubican ocho especies de aves como recurso alimenticio, siendo las familias Tinamidae, Cracidae, Odontophoridae, Phasianidae y Columbidae las más utilizadas. Las especies más citadas por los pobladores fueron: el pavo de monte $(M$. ocellata), la chachalaca $(O$. vetula) y la perdiz (C. cinnamomeus). (Figura 3 ).

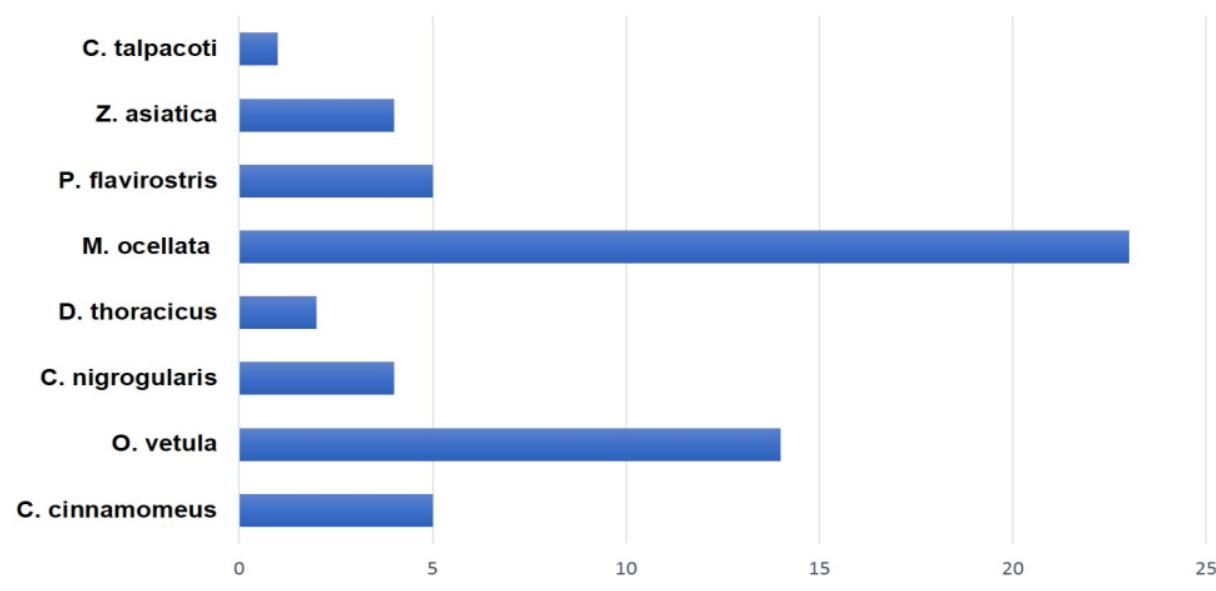

Número de veces citadas

Figura 3. Especies de aves y número de veces que fueron citadas como recurso alimenticio en la comunidad de Xanláh, Yucatán.

De acuerdo con el $43 \%$ de los encuestados, estas aves son de mayor preferencia debido a que son abundantes en la zona, poseen una mayor cantidad de carne y nutrientes y el sabor suele ser agradable. Estas características posibilitan a los pobladores prepararlas de diferentes maneras, como, por ejemplo: asadas, fritas o cocidas.

"Nos comemos la carne de la chachalaca, el pavo de monte, cada que se puede y se quiere comer, porque si no pues solo frijolitos y huevos, también pues ya no hay muchos, aunque su sabor es rico, es diferente a la de las gallinas y pavitos, da más nutrientes porque se alimentan bien" (F. Perera, entrevista personal, 2015.)

En general, las aves son cazadas por el método de espiadero (59\%), en el cual el cazador ubica su espiadero en lugares donde observa rastros del animal (excremento, plumas y restos de alimentación), generalmente en la milpa donde se encuentra los granos esparcidos en el suelo, y espera que llegue el ave y le dispara con su carabina o escopeta. Otro método es caminar por el monte o hacia la milpa, en la espera del paso de un ave.

\section{Uso pronosticador}

Con base a los encuestados $(\mathrm{N}=55)$ se registraron 13 especies de aves silvestres distribuidas en nueve familias taxonómicas para esta categoría, siendo: la familia Cracidae con una especie Ortalis vetula; la familia Cathartidae con una especie, Coragyps atratus, la familia Falconidae con una especie, Herpetotheres cachinnans, la familia Cuculidae con dos especies, Geococcyx velox y Crotophaga sulcirostris; la familia Tytonidae con una especie Tyto alba; la familia Strigidae con tres especies, Megascops guatemalae, Glaucidium brasilianum y Ciccaba virgata; la familia Vireonidae con una especie Cyclarhis gujanensis; la familia Hirundinidae con una especie Stelgidopteryx ridgwayi; y la familia Icteridae, con dos especies Dives dives y Icterus gularis. Los pobladores de Xanláh reconocen a estas aves como indicadores de eventos relacionados con el clima o temporadas, con visitas al hogar y sucesos desafortunados como enfermedades o muertes. Las vocalizaciones de algunas especies son reconocidas por los pobladores como una señal pronosticadora a dichos eventos, 
aunque en menor mención se hace referencia a la aparición de las aves en la región y al tipo de vuelo, como es señalado por tres entrevistados.

"La chachalaca cuando empieza a cantar de noche dicen que está anunciando lluvia, dicen los abuelos hay está cantando la chachalaca, están lavando sarteneja, está anunciando de que va a llover" (Graciano Pat, entrevista personal, 2015). "El kooz [(H. cachinnans)], el gavilán [Halcón] ese, pues sí, sí anuncia también lluvia, pero eso dicen los abuelos, le está doliendo sus huesos, como si tuviera un reumatismo" (L. Alcocer, entrevista personal, 2015).

Otras aves que pronostican algún evento, según el conocimiento tradicional son:

"Si la yuya [(I. gularis)] esa canta, anuncia una visita, pero tiene que tardar cantando en la casa de ese alguien, también hay uno que dicen que si canta encima de tu casa anuncia muerte, uno que hace boo boo booo como un perro, pues es también otro que dicen que pasa un pájaro blanco y grita en la casa de un recién nacido, se va a morir." (F. Perera, entrevista personal, 2015).

\section{Uso medicinal}

Solo ocho (14\%) encuestados conservan algunos conocimientos sobre el uso de aves con fines medicinales. Se identificaron ocho especies de aves que son utilizadas para aliviar padecimientos o enfermedades de origen natural y cultural (Tabla 2).

Tabla 2. Especies de aves usadas para fines medicinales en la comunidad de Xanláh, Yucatán.

\begin{tabular}{llll}
\hline Especie & Parte o producto & Forma de preparación & Padecimiento \\
\hline $\begin{array}{l}\text { Ortalis vetula } \\
\begin{array}{l}\text { Patagioenas } \\
\text { flavirostris }\end{array}\end{array}$ & Carne & Cocido & Anemia \\
\hline Zenaida asiatica & Carne & Cocido & Anemia \\
\hline Amazona xantholora & plumas & Cocido & Anemia \\
\hline Amazilia sp. & Corazón & Frotado & Protección/Absorber malas energías \\
\hline $\begin{array}{l}\text { Eumomota } \\
\text { superciliosa }\end{array}$ & Animal entero & $\begin{array}{l}\text { Quemado y preparado } \\
\text { en polvo }\end{array}$ & Traer buena suerte en el amor \\
\hline $\begin{array}{l}\text { Cyanocorax } \\
\text { yucatanicus }\end{array}$ & Animal entero & Asado & Tos \\
\hline Dives dives & Carne & Asado & Para las canas \\
\hline
\end{tabular}

\section{Uso Mascota}

El uso de aves como mascotas es poco frecuente en la localidad, ya que solo nueve encuestados (16\%) reportaron cuatro especies de aves, Columba livia, Patagioenas flavirostris, Zenaida asiatica, pertenecientes a la familia Columbidae y la Amazona albifrons de la familia, Psittacidae. Los sabedores locales mencionaron que prefieren estas aves porque son buenos animales de compañía por lo que consideran un gusto verlos en su hogar.

Según los entrevistados, las aves suelen colocarse dentro de una jaula, los cuales se les denomina como "casas" para las aves; en ellas se les coloca alimento (semillas, frutos y masa exclusivamente para los polluelos) y agua e incluso en algunos casos se les coloca percheros para que las aves duerman (Figura 4). También señalan que las especies de aves suelen ser obtenidas por compra directa y por captura en su medio natural. Las personas que se dedican a la captura de aves suelen ubicar el nido, suben al árbol y capturan el ave, posterior a ello, mantienen al polluelo bajo la alimentación de masa con agua. 


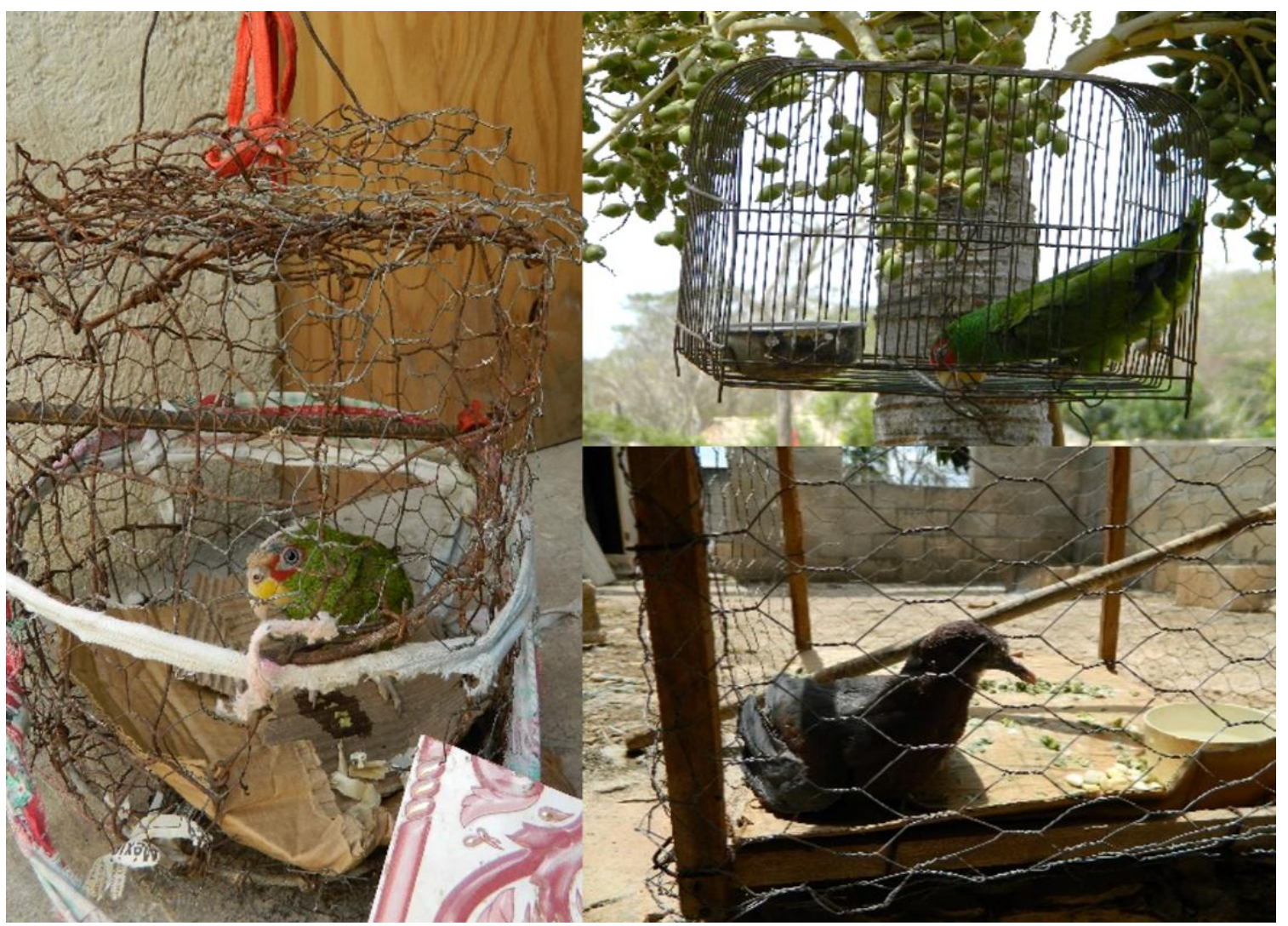

Figura 4. Jaulas rústicas donde se mantienen algunas de las aves utilizadas usadas como mascotas en la comunidad de Xanláh, Yucatán.

\section{Uso como herramienta}

Como herramienta se registró según lo reportado por el $42 \%$ de los encuestados, el uso de las plumas del pavo de monte (M. ocellata) para limpiar el hogar (sacudir el polvo) y para la elaboración de espantapájaros los cuales se colocan en el huerto y la milpa, con el fin de ahuyentar a las aves que llegan a comerse el maíz.

\section{Uso ornamental}

En cuanto a ornamento el $42 \%$ de los encuestados señaló el uso de las plumas de la cola del pavo de monte (M. ocellata) para adornar las paredes del hogar.

\section{Uso Relato}

Se reportaron dos relatos relacionados con tres especies de aves (M. ocellata, Nyctidromus albicollis y Crothopaga sulcirostris), los cuales se describen a continuación:

"Que de los pájaros más hermosos era el pavo de monte el más bonito, ya que tenía muchos colores en su cuerpo, pero era el tapacamino el que tenía envidia de eso, es por eso por lo que el tapacamino salía en las noches en busca de la belleza de las plumas del pavo de monte para robárselas y tener belleza. Por eso cuando uno sale a cazar o a caminar en la noche se encuentra uno al tapacamino en el camino porque sale a encontrarse con el pavo de monte para robarle sus plumas, por eso el tapacamino no tiene color en su cuerpo" (F. Perera, entrevista personal, 2015).

"Es secreto encontrar a las crías, si uno los ve y encuentra el nido en los martes o viernes, si uno encuentra a las crías como unos tres o cuatro, si estos son malignos o de mala suerte se convierten en víboras, pero si no, unos los ve como aves normales" ( $\mathrm{J}$. Alcocer, entrevista personal, 2015). 


\section{Índice de valor de uso (IVU) y nivel de uso significativo (UST)}

Los índices de valor de uso (IVU) calculados para todas las especies encontradas, muestran que la especie que obtuvo mayor valor de uso fue $O$. vetula con un valor de 0.65 , seguido por $M$. ocellatta con 0.49 y $T$. alba con 0.41 . Con respecto al nivel de uso significativo (UST), cuatro especies presentaron un UST superior al 20\%: O. vetula, M. ocellata, T. alba y H. cachinnans. Se registraron aves con usos específicos todos con un mismo IVU, entre ellas: C. atratus, C. talpacoti, G. velox y $M$. guatemalae (Tabla 4).

Tabla 4. Índice de Valor de Uso (IVU) y Nivel de Uso Significativo (UST) de las especies de aves registradas en la comunidad de Xanláh, Yucatán. Uso: A=Alimento, He=Herramienta, Ma=Mascota, Me= Medicinal, O= Ornamental, Pro= Pronosticador, R=Relato

\begin{tabular}{|c|c|c|c|c|}
\hline Especie & Usos & $\begin{array}{l}\text { Índice de Valor } \\
\text { de Uso (IVU) }\end{array}$ & $\begin{array}{l}\mathrm{N}^{\circ} \text { de menciones } \\
\text { (Frecuencia) }\end{array}$ & $\begin{array}{c}\text { Nivel de Uso } \\
\text { Significativo (UST) } \\
\%\end{array}$ \\
\hline $\begin{array}{l}\text { Crypturellus } \\
\text { cinnamomeus }\end{array}$ & Alimento & 0.09 & 5 & $9 \%$ \\
\hline Ortalis vetula & $\begin{array}{l}\text { Alimento, Medicinal, } \\
\text { Pronosticador }\end{array}$ & 0.65 & 32 & $\mathbf{5 8 \%}$ \\
\hline Colinus nigrogularis & Alimento & 0.07 & 4 & $7 \%$ \\
\hline Dactylortyx thoracicus & Alimento & 0.03 & 2 & $4 \%$ \\
\hline Meleagris ocellata & $\begin{array}{l}\text { Alimento, Relato, } \\
\text { Herramienta, Ornamental }\end{array}$ & 0.49 & 23 & $42 \%$ \\
\hline Coragyps atratus & Pronosticador & 0.01 & 1 & $2 \%$ \\
\hline $\begin{array}{l}\text { Herpetotheres } \\
\text { cachinnans }\end{array}$ & Pronosticador & 0.21 & 12 & $22 \%$ \\
\hline $\begin{array}{l}\text { Columba } \\
\text { livia }\end{array}$ & Mascota & 0.03 & 2 & $4 \%$ \\
\hline Patagioenas flavirostris & $\begin{array}{l}\text { Alimento, Mascota, } \\
\text { Medicinal }\end{array}$ & 0.12 & 6 & $11 \%$ \\
\hline Zenaida asiatica & $\begin{array}{l}\text { Alimento, Mascota, } \\
\text { Medicinal }\end{array}$ & 0.10 & 6 & $11 \%$ \\
\hline Columbina talpacoti & Alimento & 0.01 & 1 & $2 \%$ \\
\hline Amazona albifrons & Mascota & 0.07 & 4 & $7 \%$ \\
\hline Amazona xantholora & Medicinal & 0.03 & 2 & $4 \%$ \\
\hline Geococcyx velox & Pronosticador & 0.01 & 1 & $2 \%$ \\
\hline Crotophaga sulcirostris & Relato Pronosticador & 0.03 & 2 & $4 \%$ \\
\hline Tyto alba & Pronosticador & 0.41 & 23 & $42 \%$ \\
\hline Megascops guatemalae & Pronosticador & 0.01 & 1 & $2 \%$ \\
\hline $\begin{array}{l}\text { Glaucidium } \\
\text { brasilianum }\end{array}$ & Pronosticador & 0.09 & 5 & $9 \%$ \\
\hline Ciccaba virgata & Pronosticador & 0.09 & 5 & $9 \%$ \\
\hline Nyctidromus albicollis & Relato & 0.01 & 1 & $2 \%$ \\
\hline Amazilia sp. & Medicinal & 0.01 & 1 & $2 \%$ \\
\hline Eumomota superciliosa & Medicinal & 0.01 & 1 & $2 \%$ \\
\hline $\begin{array}{l}\text { Cyanocorax } \\
\text { yucatanicus }\end{array}$ & Medicinal & 0.01 & 1 & $2 \%$ \\
\hline Cyclarhis gujanensis & Pronosticador & 0.07 & 4 & $7 \%$ \\
\hline Stelgidopteryx ridgwayi & Pronosticador & 0.03 & 2 & $4 \%$ \\
\hline Dives dives & Pronosticador & 0.03 & 2 & $4 \%$ \\
\hline Icterus gularis & Pronosticador & 0.07 & 4 & $7 \%$ \\
\hline
\end{tabular}




\section{DISCUSIÓN}

En este estudio se encontró que las aves sirven como recurso alimenticio o incluso como elementos complementarios y/o alternativos en la alimentación de los pobladores, como también fue reportado por García et al. (2017) en su estudio etnozoológico de las aves y mamíferos silvestres asociados a huertos frutícolas de Zacualpan de Amilpas, Morelos, México donde se reconoce al uso alimenticio como uno de los más significativos.

De las aves registradas con un uso alimenticio, las especies preferidas destacan por ser especies abundantes y cercanas en la región, además de ser especies que forman parte de la dieta habitual de la comunidad, como también es señalado por Santos-Fita et al. (2012) quién observaron que las especies frecuentemente cazadas son aves de gran tamaño, razón por la cual, proporcionan una biomasa mayor que otras especies de aves. De hecho, Lira et al. (2014) en su estudio sobre el uso y aprovechamiento de la fauna silvestre en la selva zoque, Chiapas, México, señalan que el grupo biológico más cazado después de los mamíferos, son las aves.

Los resultados obtenidos en este estudio siguen esta tendencia, ya que las especies preferidas por los cazadores son la chachalaca $(O$. vetula), el pavo de monte $(M$. ocellata) y la perdiz $(C$. cinnamomeus) debido a que poseen una mayor cantidad de carne y nutrientes y el sabor suele ser agradable, posibilitando a los pobladores prepararlas de diferentes maneras, como, por ejemplo: asadas, fritas o cocidas. Caso similar ocurre con las aves registradas de la familia Columbidae, las cuales son constantemente cazadas por ser una fuente adicional de proteínas, además de ser aves que se capturan fácilmente por su actividad de forrajeo a nivel del suelo (NÓBREGA et al., 2011; GALVAGNE-LOSS et al., 2014).

Las aves juegan un rol importante para la comprensión de eventos o presagios culturales y/o naturales como sobrenaturales. De las aves registradas como pronosticadoras de lluvia destaca la chachalaca $(O$. vetula) y el koozt (H. cachinnans) que ya se han reportado por Galvagne-Loss et al. (2013), ya que se cree que el canto repetitivo de ambas especies pronostica la lluvia.

En cuanto al canto de la yuya (I. gularis), éste indica una posible visita de familiares y/o amigos al hogar, esta ave igual ha sido reportada por Alves et al. (2013) pero con un distinto presagio; el de atraer eventos desagradables. De acuerdo con este mismo autor, las vocalizaciones de las aves son a menudo consideradas como presagios de origen natural o acontecimientos sobrenaturales (adivinaciones ornitológicas) y son clasificadas de acuerdo a los eventos predichos, sin embargo, Silveira (2010) y Guerrero et al. (2010) observaron que existen otras señales, tales como: la elaboración y construcción de nidos, el vuelo y la aparición de parvadas de golondrinas Stelgidopteryx ridgwayi como lo reportado en la comunidad de Xanláh.

Con respecto a los miembros de las familias Strigidae y Tytonidae, estos están fuertemente relacionados con sucesos desagradables e incluso como pronosticadores de muerte (NUÑEZ-GARCÍA et al., 2012), lo que se constata en el presente estudio, donde se observa que el canto de las especies Tyto alba (kooka en maya), Glaucidium brasilianum (tooka en maya) y Ciccaba virgata (boo-ka en lengua maya) son indicadores de mal augurio y suelen asociarse con la muerte de algún familiar.

Por lo que se refiere al uso alternativo de aves con fines medicinales, solo se registró el uso de ocho especies, las cuales son usadas principalmente para contrarrestar principios de anemia y el desarrollo de las canas. Este resultado contrasta con el trabajo de Retana-Guiascón et al. (2014) desarrollado en comunidades mayas de Campeche. Es decir, en dicho estudio, el número de especies asciende a 13 e incluye el uso de la paloma ( $P$. flavirostris) y el loro (A. xantholora). Otra especie reportada como ave medicinal es el colibrí: Amazilia sp., la cual ha sido muy usada en diversos estados del país (NÚÑEZ-GARCÍA et al., 2012; GUERRERO-ORTIZ, 2013; JAIMES-YESCAS et al., 2014) principalmente para tener buena suerte en el amor, como el caso reportado en este estudio, el cual se emplea para atraer el amor de la persona deseada.

De acuerdo con Alonso-Castro (2014), alrededor del 35\% de la fauna es usada con fines medicinales, siendo las aves el grupo taxonómico más citado. De esta manera los animales utilizados como medicina tradicional, son recursos importantes ya que mantienen el vínculo entre la población con el medio ambiente y su uso promueve el saber tradicional asociado a ellos (ALVES y ROSA, 2013).

Con respecto a las aves que se usan como mascotas, las especies registradas de manera más frecuente por los pobladores de Xanláh son las pertenecientes a las familias Columbidae y Psitacidae. Estos datos coinciden con otros estudios relacionados con el uso de aves silvestres, donde se demuestra con evidencias la preferencia popular por estas aves con relación a otros grupos de aves 
(BEZERRA et al., 2011, 2012; ALVES et al., 2013; LIRA-TORRES et al., 2014; ROLDÁN-CLARA, et al., 2014). Retana-Guiascón et al. (2014) enfatizan que las comunidades mayas hacen uso frecuente de las aves como mascotas, siendo esta una forma de mantener su relación y permanencia con la naturaleza.

El uso ornamental, principalmente de las plumas de la cola del pavo de monte, está asociado generalmente con los cazadores, los cuales aprovechan los derivados del recurso alimenticio y exponen las plumas como adorno. Este uso no había sido reportado, ya que para la región yucateca solo están reportados usos ornamentales de astas de venados y pieles de mamíferos (RETANAGUIASCÓN et al., 2014).

El único registró que se obtuvo como herramienta, son las plumas del pavo de monte usadas principalmente por las mujeres del hogar como plumeros o sacudidor de polvo, que, aunque no suelen ser empleadas con frecuencia cubren con su finalidad de uso, razón por la cuál es de importancia cultural para la población.

El relato representativo de la coloración de las plumas del pavo de monte ( $M$. ocellata) y el tapacamino ( $N$. albicollis), señalado en las entrevistas, es el conocimiento relatado de aquella interacción de un campesino con la naturaleza, al igual que el relato donde se incluye al garrapatero pijuy (C. sulcirostris). Esta relación coincide con el trabajo de Tidemann y Whiteside (2010) quienes señalan que los relatos con representaciones de aves contienen información biológica, siendo expresadas de diferentes formas, indicando a menudo características de plumaje, hábitat, comportamientos, alimentación, así como la relación de las aves con la tierra.

Con respecto a los valores IVU, las especies con mayor importancia cultural son la chachalaca (O. vetula) y el pavo de monte (M. ocellata), por lo tanto, poseen un alto valor de uso y se sitúan en la preferencia de los pobladores, dado que cubren principalmente su necesidad alimenticia y a su vez suelen ser empleadas para diversos usos (medicinal, herramienta, pronosticadora, etc.), por lo que son consideradas importantes a nivel comunitario, esto es similar a los resultados reportados por Cortés y Chuprine (2018) quienes señalan el índice de importancia cultural del uso del grupo taxonómico en su estudio, fueron las aves, que reportando un uso múltiple (alimenticio, medicinal,vestimenta, así como para las actividades rituales. Estos resultados pueden deberse a lo mencionado por Alves (2012) quien propone que una sola especie puede ser utilizada de diferentes maneras y para diferentes fines en función de los aspectos culturales asociados, así como el uso propuesto. También destaca el alto valor de uso de la especie T. alba, ya que su uso (pronosticador) ha sido el más frecuente y el más mencionado por los pobladores de la comunidad, por lo que los conocimientos tradicionales en torno a esta especie recaen fuertemente sobre el vínculo de asociar un ave nocturna con la muerte.

El conocimiento cultural de las aves en el poblado de Xanláh es un reflejo del constante uso determinado de cada especie de ave silvestre, el cual se observa en las cuatro especies (O. vetula, M. ocellata, T. alba y H. cachinnans) altamente valoradas en la comunidad, las cuales presentan un UST superior al 20\%. De acuerdo con Londoño-Betancourth (2012), la valoración cultural de la fauna es el resultado de la importancia y los usos que los individuos y la comunidad le dan en función a los beneficios proporcionados, de modo que, las especies con mayor aceptación cultural tendrán un mayor número de usos en comparación con las especies de menor aceptación cultural, por lo cual es importante valorizar los usos comunitarios de las aves.

\section{CONCLUSIONES}

Se registraron 27 especies de aves con siete categorías de uso: alimenticio, pronosticador, medicinal, mascota, herramienta, ornamental y relato, siendo los representantes de las familias Cracidae, Phasianidae y Columbidae los más usados.

El principal uso de la avifauna es el pronosticador, con trece especies de aves. Las especies más mencionadas son Ortalis vetula, Tyto alba y Herpetotheres cachinnans.

Se registró un total de ocho especies con uso alimenticio, destacando el pavo de monte (Meleagris ocellata) y la chachalaca (Ortalis vetula) con alta frecuencia de mención.

Las especies con altos valores con respecto al uso significativo fueron nuevamente Ortalis vetula (0.65) y Meleagris ocellata (0.49), así como Tyto alba (0.41) por lo que son consideradas importantes a nivel comunitario.

Se identificaron cuatro especies con un UST superior al 20\% (Ortalis vetula, Meleagris ocellata, Tyto alba y Herpetotheres cachinnans) las cuales son altamente valoradas en la comunidad. Las especies de menor aceptación cultura obtuvieron un UST del $2 \%$ entre ellas Coragyps atratus, 
Columbina talpacoti, Geococcyx velox y Megascops guatemalae.

\section{LITERATURA CITADA}

ALONSO-CASTRO, A. Use of medicinal fauna in Mexican traditional medicine. Journal of ethnopharmacology 152:53-70, 2004

ALVES, R. Y SOUTO, W. Ethnozoology. A brief introduction. Ethnobiology and Conservation 4:1-13, 2015.

ALVES, R. Y ROSA, I. Introduction: Toward a Plural Approach to the Study of Medicinal Animals. En: Alves, R. y Rosa, I. (Edit) Animals in Traditional Folk Medicine. Implications for Conservation. 2013. Springer. Nueva York. E.U.A. pp: 1-9.

ALVES, R., LOURENCO, R., SOUTO, W., BEZERRA, M. Y LOURDES-RIBEIRO, A. Ethnoornithology and conservation of wilds birds in the semi-arid Catinga of northeastern Brazil. Journal of Ethnobiology 9:1-12, 2013.

ALVES, R.,SOUSA, R., BRITO, D., BARBOSA, J., BARROS, A. Y PERERIRA, T. Traditional uses of medicinal animals in the semi-arid region of northeastern $\quad$ Brazil. Journal of Ethnobiology and Ethnomedicine 8: 1-7, 2012.

ANTONIO, N. Saberes con rostros de mujeres indígenas, conocimientos tradicionales y actividades productivas, en el ejido El Remolino, Municipio de Huitiupán, Chiapas. 2012, p.121. Tesis de Licenciatura. Universidad intercultural de Chiapas. San Cristobal de Las Casas, México.

BALBUENA JARA, Oscar. Metodología y rapport. El monitoreo, el seguimiento y la investigación social en ámbitos de intervención del mecanismo nacional de prevención de la tortura. Revista estudios paraguayos (CEADUC-UC), [S.1.], p. 277-300, 2017. Disponible en: $<$ http://epy.dreamhosters.com/index.php/RESPY/article/view/56>.

BARROS, F., VARELA, S., PEREIRA, H. Y VICENTE, L. Medicinal use of fauna by traditional community in the Brazilian Amazonia. Journal of Ethnobiology and Ethnomedicine 8: 1-19, 2012.

BEZERRA D. DE ARAUJO H. Y ALVES R. The use of wild birds by rural communities in the semi-arid region of Rio Grande do Norte State, Brazil. Bioremediation, Biodiversity and Bioavailability 1:117-120, 2011.

BEZERRA, D., DE ARAUJO, H. Y ALVES, A. Birds and people in semiarid northeastern Brazil: symbolic and medicinal relationship. Journal of 2012.

Ethnobiology and Ethnomedicine 9:1-11,

CORTÉS-SUÁREZ, J. E. y A. CHUPRINE-VALLADARES. Usos de la fauna silvestre por los Ngäbe de la Península de Osa, Costa Rica. Ethnoscientia 3: 1-14. 2018

CHABLÉ, J. y H. DELFÍN. Uso tradicional de fauna silvestre. En: Durán R. y M. Méndez (Eds.). Biodiversidad y desarrollo Humano en Yucatán. CICY, PPM-FMAM, CONABIO. SEDUMA. pp: 377-381, 2010.

MORGA, L.E. Teoría y Técnicas de la Entrevista. Editorial RED TERCER MILENIO S.C. 2012. Estado de México. 99p. Disponible en: http://www.aliat.org.mx/BibliotecasDigitales/salud/Teoria_y_tecnica_de_la_entrevista. pdf

DE LA GARZA Mercedes. Aves Sagradas de los Mayas. Centro de Estudios Mayas del Instituto de Investigacionnes Filológicas, UNAM. 1995. México. 157p.

DUNN, J. Y J. ALDERFER. National geographic Field guide to the birds of Eastern North america. 2008. Washington, E.U.A. 431 pp.

FERRO, M. Guías de observación etnográfica y valoración cultural: Santuarios y oficios. Apuntes 23:56-69, 2010. Disponible en: http://www.scielo.org.co/pdf/apun/v23n1/v23n1a06.pdf

FLORES, J. Y I. ESPEJEL. Tipos de vegetación de la península de Yucatán. Etnoflora yucatanenese, Fasciculo 3. Universidad Autónoma de Yucatán y Sostenibilidad Maya, 1994.

GALVAGNE-LOSS, A., E. COSTA-NETO Y F. FLORES. Ornitoáugures no povoado de Pedra Branca, Santa Teresinhia, estado da Bahia, Nordeste do Brasil. Etnobiología 11(3):45-53, 2013.

GARCÍA, E. Modificaciones al sistema de clasificación climática de Köppen. Instituto de Geografía. Universidad Nacional Autónoma de México, 19787. México. 90 p.

GARCÍA-F. A.; MOJICA-PEDRAZA, S.; BARRETO-SÁNCHEZ, S. D.; MONROY-ORTIZ, C.; MONROY-MARTÍNEZ, R. Estudio etnozoológico de las aves y mamíferos silvestres asociados a huertos frutícolas de Zacualpan de Amilpas, Morelos, México. Revista de Ciencias Ambientales, Heredia, v. 51, n. 2, p. 110-132, 2017. 
GÖTZ C. M. Caza y pesca prehispánicas en la costa norte peninsular yucateca. Ancient Mesoamerica, 23, pp 421-439. 2012. doi:10.1017/S0956536112000272. Disponible en: http://journals.cambridge.org/abstract_S0956536112000272

GONZÁLEZ H.R; CHABLÉ- S.J; AGUILAR C.W; MANRÍQUE S. P. El comercio de aves silvestres en la ciudad de Mérida, Yucatán, México. Revista Ecosistemas y Recursos Agropecuarios. 3(9):379-389. Universidad Juárez Autónoma de Tabasco, México. 5(14):271-281. 2018. DOI: $10.19136 /$ era.a5n14.1242

GONZÁLEZ, T. Lo animal en la cosmovisión mexica o mesoamericana. En: González, T. (Ed.) Animales y plantas en la cosmovisión mesoamericana. Plaza y Valdés. CONACULTA-INAH y Sociedad Mexicana para el Estudio de Las Religiones. 2001. México, D.F. pp: 107-122.

GUBER, R. El salvaje metropolitano. Reconstrucción del conocimiento social en el trabajo de campo. Paidós Estudios de comunicación. Buenos Aires, Argentina. 220 p., 2005.

GUERRERO, M., G. SERRANO Y V. SERRANO. Aves con atributos pronosticadores, medicinales y mágico-religiosos entre los tojolabales (tojol winik'otik) del ejido saltillo, las margaritas, Chiapas. El canto del Centzontle 1(2): 190-203, 2010.

GUERRERO-ORTIZ, S. Uso medicinal de la fauna silvestre por indígenas tlahuicas en Ocuilan, México. 2013, 78 p. Tesis de Licenciatura. Universidad Nacional Autónoma de México, México.

HOWELL, S. Y S. WEBB. A Guide to the Birds of Mexico and Northern Central America. New York: Oxford University Press, 1995.

INEGI. Censo de Población y Vivienda 2010. Principales resultados por localidad. (ITER). Disponible en: http://www.inegi.gob.mx. 2010.

JAIMES-YESCAS M., G. GÓMEZ ÁLVAREZ., N. PACHECO Y S. REYES . Uso y manejo de la avifauna en San Miguel Tzinacapan, municipio de Cuetzalan del Progreso, Puebla, México. En: Vásquez-Dávila, M. A. (Ed.): Aves, personas y culturas. Estudios de Etnoornitología 1. CONACYT/ITVO/Carteles Editores/UTCH. Oaxaca, México. 2014, pp: 243-259.

KAUFMAN, K. Guía de Campo de las Aves de Norteamérica. Houghton Mifflin Company. New York, 2005.

LIRA-TORRES, I., M. BRIONES-SALAS., F. GÓMEZ DE ANDA., D. OJEDA-RAMIREZ Y A. PELÁEZ. Uso y aprovechamiento de la fauna silvestre en la selva zoque, México. Acta Zoológica Mexicana 30:74-90, 2014.

LÓPEZ-MEDELLÍN, X. Y E. ÍÑIGO. La captura de aves silvestres en México: Una tradición milenaria y las estrategias para regularla. CONABIO. Biodiversitas 83:11-15, 2009.

Londoño-Betancourth, J. Valoración cultural del uso e importancia de la fauna silvestre en cautividad en tres barrios de Pereira (Risaralda). Boletín científico. Museo de Historia Natural 13:33-46, 2012.

MÉNDEZ-CABRERA, F. Y S. MONTIEL. Diagnostico preliminar de la fauna y flora silvestre utilizada por la población maya de dos comunidades costeras de Campeche, México. Universidad y Ciencia. 23(2):127-139, 2007.

NARANJO, E., J. LÓPEZ-ACOSTA Y R. DIRZO. La cacería en México. Biodiversitas 91:6-10, 2010.

NAVARIJO, O. Ma. L. Las aves en el mundo maya prehispánico. En STAINES, C.L. (Coord.) La pintura mural prehispánica en México. II Area Maya. Tomo III Estudio. 2001. UNAM-México (pp. 221-253)

NÓBREGA V., J. BARBOSA Y R. ALVES. Utilização de aves silvestres por moradores do município de Fagundes, Semiárido paraibano: uma série Ciências Biológicas 11(2):165- 175., 2011. abordagem etnoornitológica. Sitientibus

NÚÑEZ-GARCÍA, R., M. FUENTE-CARRASCO Y C. VENEGAS -BARRERA. La avifauna en la memoria biocultural de la juventud indígena en la sierra Juárez de Oaxaca, México. Universidad y Ciencia 28 (3):201-216, 2012.

LIRA-TORRES, I; BRIONES-SALAS, M; GÓMEZ DE ANDA, F, OJEDA-RAMÍREZ, D; PELÁEZ ACERO, A. Uso y aprovechamiento de la fauna silvestre en la selva zoque, México. Acta Zoológica Mexicana (nueva serie), 30 (1), 2014. Disponible en: https://www.redalyc.org/articulo.oa?id=575/57530109006

PETERSON, T. Y E. CHALIF. Aves de México Guía de Campo. Editorial Diana. 1989. México. 
$473 \mathrm{p}$.

PUC-GIL, R. Y O. RETANA-GUIASCÓN. Uso de la fauna silvestre en la comunidad maya de Guadalupe, Campeche, México. Etnobiología 10(2):1-11, 2012.

REYES-GARCÍA, V. Conocimiento ecológico tradicional para la conservación: dinámicas y conflictos. Papeles de relaciones ecosociales y cambio global 107:39-55, 2009.

RETANA-GUIASCÓN, O., R. PUC-GIL Y L. MARTÍNEZ-PECH. Uso de la fauna silvestre por comunidades mayas de Campeche, México: el caso de las aves. En: Vásquez-Dávila M, A. (Ed.): Aves, personas y culturas. Estudios de Etno-ornitología 1. CONACYT/ITVO/Carteles Editores/UTCH. 2014. Oaxaca, México. pp: 35-46.

RETANA-GUIASCÓN, O., M. AGUILAR-NAH Y G. NIÑO-GÓMEZ. Uso de la vida silvestre y alternativas de manejo integral. El caso de la comunidad Maya de Pich, Campeche, México. Tropical and Subtropical Agroecosystems 14:885-890, 2011.

ROLDÁN-CLARA, B., X. LÓPEZ-MEDELLÍN., I. ESPEJEL Y E. ARELLANO. Literature review of the use of birds as pets in Latin-America, with a detailed perspective on Mexico. Ethonobiology and conservation 3:1-18, 2014.

RUSELL, H. Métodos de investigación en Antropología. Abordajes cualitativos y cuantitativos. AltaMira Press. 1995. California. E.U.A. 438 p.

SANTOS- FITA, D., E. NARANJO Y J. RANGEL-SALAZAR. Wildlife uses and hunting patterns in rural comunities of the Yucatan Peninsula, Mexico. Journal of Ethnomedicine 8:1-17, 2012.

SIBLEY, D. The Sibley guite to birds. Alfred A Knopf. New York, E.U.A, 2000.

SILVEIRA, R. Conhecimiento Ecológico Tradicional de Aves da Comunidade Cuiabá Miriam, Pantanal de Mata Grosso. 2010. Universidade do estado de Mato Grosso. Cáceres, MSc diss.

TIDEMANN, S. Y T. Whiteside. Aboriginal Stories: Th Rich and Colour of Australian Birds. En: Tidemann, S. y A. Gosler. (Eds.). Ethno-ornithology. Birds and Indigenous people, Culture and Society. Earthscan. 2010. Washington. DC. pp:154-170.

TOSCANO, J. Uso tradicional de plantas medicinales en la vereda San Isidro, municipio de San José de Pare-boyacá: un estudio preliminar usando técnicas cuantitativas. Acta Biol. Colomb.11(2):137-46, 2006.

TOZZER A.M y ALLEN G.M. Animal Figures in the Mayan Codices. Vol. IV. No.3. Cambridge Mass. Published by Museum. 1910. 180p. Disponible en: https://archive.org/details/animalfiguresinm00tozzrich/page/n2

UC K. M. Ideas y prácticas sobre las aves entre los niños de Xcunyá, $\quad$ Yucatán. 2014. Tesis de Maestría. Centro de Investigación y de Estudios Avanzados del Instituto Politécnico Nacional. Unidad Mérida. Departamento de Ecología Humana. Mérida, Yucatán. 131 p.

UC K.M. y M.D. CERVERA. ¡Vamos a pescar!: Los niños mayas y las aves de Yucatán, México. En: Vásquez-Dávila, M. A. (Ed.): Aves, personas y culturas. Estudios de Etno-ornitología 1. CONACYT/ ITVO/Carteles Editores/UTCH. . 2014. Oaxaca, México. P. 19-34. Disponible en: https://www.mda.cinvestav.mx/Portals/0/Maestria/productividad/Uc2014.pdf?ver=201 905-23-110713-493 